Research Article; Received: October 10, 2020; Accepted: June 10, 2021

\title{
AN EFFICIENT VARIANT OF DUAL TO PRODUCT AND RATIO ESTIMATORS IN SAMPLE SURVEYS
}

\author{
Sayed Mohammed ZEESHAN ${ }^{1}$, Gajendra K. VISHWAKARMA ${ }^{2}$, and Manish KUMAR ${ }^{3}$ \\ ${ }^{1}$ School of Advanced Sciences and Languages, Vellore Institute of Technology, \\ Bhopal-466114, INDIA \\ ${ }^{2}$ Department of Mathematics \& Computing, Indian Institute of Technology \\ (ISM), Dhanbad-826004, INDIA \\ ${ }^{3}$ Department of Agricultural Statistics, Acharya Narendra Deva University of \\ Agriculture \& Technology, Ayodhya-224229, INDIA
}

\begin{abstract}
This manuscript considers a dual to product and ratio estimator for estimating the finite population mean of study variable on applying a simple transformation to the auxiliary variable by using its average values in the population that is generally available in practice. The mean square error (MSE) of the proposed estimator has been obtained to the first degree of approximation. The optimum values and range of suitably chosen scalar, under which the proposed estimator perform better, have been determined. A method to lower the MSE of the proposed estimator relative to that of the MSE of the linear regression estimator is developed for small sample sizes. Theoretical and empirical studies have been done to demonstrate the superiority of the proposed estimator over the other estimators.
\end{abstract}

\section{INTRODUCTION}

There are numerous number of ratio and product type estimators available in survey literature from the time ratio estimator was developed by Cochran [4], and the product estimator was defined by Robson $[12$ that was revisited by Murthy [1]. Ratio and product type estimators have been largely used due to computational simplicity, greater applicability to the general design and researchers' impulsive draw towards it. Most of the ratio and product type estimators recently developed are simply a modification of other existing estimators available in the literature.

2020 Mathematics Subject Classification. Primary 62D05.

Keywords. Study variable, auxiliary variable, population mean, Bias, MSE.

■ zeeshan008x52@gmail.com; vishwagk@rediffmail.com-Corresponding author; manishstats88@gmail.com

(D) 0000-0002-8454-8633; 0000-0002-2804-4334; 0000-0002-4989-1612. 
This has led to the accumulation of a large number of the ratio as well as product type estimators with cumbersome structure over the time. Often these estimators require the knowledge of other population parameters in advance or has to guess it with the experience gathered over the period of time in sample survey or estimate it through pilot survey or the sample itself and in optimum case the MSE of the proposed estimator is found generally equivalent to the MSE of the regression estimator. Moving in this direction, we have proposed the dual to product and ratio estimators and shown that how in optimal case their minimum MSE becomes nothing but MSE of regression estimator. We have carried out then the key study of developing the new estimator using the previously proposed dual to product and ratio estimators which will be called parent estimator for the newly developed estimator. The new estimator' MSE is improved to an extent that it becomes better or more efficient than the regression estimator. One more aspect of our method is the important role played by the bias of the estimator in improving MSE which was neglected before in the survey literature works in the area of ratio and product estimators.

Let $U=\left\{U_{1}, U_{2}, \ldots, U_{N}\right\}$ be a finite population of size N. Also, let $Y$ and $X$ be the study and auxiliary variables, respectively, taking the values $y_{i}$ and $x_{i}$ on the $i^{\text {th }}$ unit $U_{i}(i=1,2, \ldots, N)$ of the population $U$. Assuming that the population mean $\bar{X}$ of the auxiliary variable $X$ is known,the population mean $\bar{Y}$ of the study variable $Y$ is estimated by selecting a sample of size $n$ (with $n<N$ ) from the population $U$ using simple random sampling without replacement (SRSWOR) scheme.

The ratio estimator of $\bar{Y}$ as developed by Cochran [4, and the product estimator of $\bar{Y}$ as developed by Murthy [11 are given, respectively, by

$$
\begin{aligned}
& \bar{y}_{R}=\bar{y}\left(\frac{\bar{X}}{\bar{x}}\right) \\
& \bar{y}_{P}=\bar{y}\left(\frac{\bar{x}}{\bar{X}}\right)
\end{aligned}
$$

with their respective Biases and MSEs to the first order of approximations as

$$
\begin{gathered}
\operatorname{Bias}\left(\bar{y}_{R}\right)=\lambda \bar{Y}\left[C_{x}{ }^{2}-\rho_{y x} C_{y} C_{x}\right] \\
\operatorname{Bias}\left(\bar{y}_{P}\right)=\lambda \bar{Y} \rho_{y x} C_{y} C_{x} \\
M S E\left(\bar{y}_{R}\right)=\lambda \bar{Y}^{2} C_{y}^{2}\left[1+\left(\frac{C_{x}}{C_{y}}\right)^{2}-2 \rho_{y x} \frac{C_{x}}{C_{y}}\right] \\
\operatorname{MSE}\left(\bar{y}_{P}\right)=\lambda \bar{Y}^{2} C_{y}^{2}\left[1+\left(\frac{C_{x}}{C_{y}}\right)^{2}+2 \rho_{y x} \frac{C_{x}}{C_{y}}\right]
\end{gathered}
$$


where $\bar{y}=\frac{1}{n} \sum_{i=1}^{n} y_{i}$ and $\bar{x}=\frac{1}{n} \sum_{i=1}^{n} x_{i}$ are the sample means of $Y$ and $X$, respectively. Also, $C_{y}$ and $C_{x}$ represent the coefficients of variations of the variables $Y$ and $X$, respectively. Moreover, $\rho_{y x}$ denotes the correlation coefficient between the study variable $Y$ and the auxiliary variable $X$. The notations used above are as follows:

$$
\begin{gathered}
\lambda=\frac{1-f}{n}, f=\frac{n}{N}, C_{y}^{2}=\frac{S_{y}^{2}}{\bar{Y}^{2}}, C_{x}^{2}=\frac{S_{x}^{2}}{\bar{X}^{2}}, \rho_{y x}=\frac{S_{y x}}{S_{y} S_{x}} \\
S_{y}^{2}=\frac{1}{(N-1)} \sum_{i=1}^{N}\left(y_{i}-\bar{Y}\right)^{2}, S_{x}^{2}=\frac{1}{(N-1)} \sum_{i=1}^{N}\left(x_{i}-\bar{X}\right)^{2} \\
S_{y x}=\frac{1}{(N-1)} \sum_{i=1}^{N}\left(y_{i}-\bar{Y}\right)\left(x_{i}-\bar{X}\right)
\end{gathered}
$$

The classical linear regression estimator for population mean $\bar{Y}$ is defined by

$$
\hat{\bar{Y}}_{\text {reg }}=\bar{y}+b_{y x}(\bar{X}-\bar{x})
$$

where $b_{y x}$ is the sample regression coefficient of $Y$ on $X$.

Also, the Bias and MSE of $\hat{\bar{Y}}_{\text {reg }}$ to the first order of approximations are given, respectively, by

$$
\begin{gathered}
\operatorname{Bias}\left(\hat{\bar{Y}}_{\text {reg }}\right)=-\operatorname{cov}\left(\bar{x}, b_{y x}\right) \\
\operatorname{MSE}\left(\hat{\bar{Y}}_{\text {reg }}\right)=\lambda \bar{Y}^{2} C_{y}^{2}\left(1-\rho_{y x}^{2}\right)
\end{gathered}
$$

Srivenkataramana [15] and Bandyopadhya [2] suggested a dual to ratio and a dual to product estimators, respectively, for $\bar{Y}$ as

$$
\begin{aligned}
& \bar{y}_{R}^{*}=\bar{y}\left(\frac{\bar{x}^{*}}{\bar{X}}\right) \\
& \bar{y}_{P}^{*}=\bar{y}\left(\frac{\bar{X}}{\bar{x}^{*}}\right)
\end{aligned}
$$

with their respective Biases and MSEs to the first order of approximations as

$$
\begin{gathered}
\operatorname{Bias}\left(\bar{y}_{R}^{*}\right)=-g \lambda \bar{Y} \rho_{y x} C_{y} C_{x} \\
\operatorname{Bias}\left(\bar{y}_{P}^{*}\right)=\lambda \bar{Y}\left[g^{2} C_{x}^{2}+g \rho_{y x} C_{y} C_{x}\right] \\
\operatorname{MSE}\left(\bar{y}_{R}^{*}\right)=\lambda \bar{Y}^{2} C_{y}^{2}\left[1+g^{2}\left(\frac{C_{x}}{C_{y}}\right)^{2}-2 g \rho_{y x} \frac{C_{x}}{C_{y}}\right]
\end{gathered}
$$




$$
\operatorname{MSE}\left(\bar{y}_{P}^{*}\right)=\lambda \bar{Y}^{2} C_{y}^{2}\left[1+g^{2}\left(\frac{C_{x}}{C_{y}}\right)^{2}+2 g \rho_{y x} \frac{C_{x}}{C_{y}}\right]
$$

where $\bar{x}^{*}=(1+g) \bar{X}-g \bar{x}$ is an unbiased estimator of $\bar{X}$, and $g=n /(N-n)$.

Some recent developments towards the formulation of different classes of dual to product-cum-dual to ratio estimators have been made by Singh et al. 13, and Choudhury and Singh [3]. Moreover, Adebola et al. [?] developed a class of regression estimator with cum-dual ratio estimator as intercept. The recently developed estimators as described here are listed in Table 1.

TABLE 1. Recent developed estimators of $\bar{Y}$

\begin{tabular}{|c|c|}
\hline Authors & Estimators \\
\hline Singh et al. 13 & $\bar{y}_{P R}^{*}=\eta \bar{y}\left(\frac{a \bar{X}+b}{a \bar{x}^{*}+b}\right)+(1-\eta) \bar{y}\left(\frac{a \bar{x}^{*}+b}{a \bar{X}+b}\right)$ \\
\hline Choudhury and Singh 3 & $\bar{y}_{C S}^{*}=\bar{y}\left[\alpha \frac{\bar{x}}{\bar{x}^{*}}+(1-\alpha) \frac{\bar{x}^{*}}{X}\right]$ \\
\hline Adebola et al. [?] & $\bar{y}_{R d}^{*}=\bar{y} \frac{\bar{x}^{*}}{\bar{X}}+\alpha\left(\bar{X}-\bar{x}^{*}\right)$ \\
\hline
\end{tabular}

In Table 1, $\eta, a, b$ and $\alpha$ denote the scalars, which are suitably determined so as to minimize the MSEs of the concerned estimators. Also, the expressions for the Biases and MSEs of various estimators to the terms of order $o\left(n^{-1}\right)$ are given by

$$
\begin{gathered}
\operatorname{Bias}\left(\bar{y}_{P R}^{*}\right)=\lambda \bar{Y}\left[\eta\left(\frac{a \bar{X}}{a \bar{X}+b}\right)^{2} g^{2} C_{x}^{2}+(2 \eta-1)\left(\frac{a \bar{X}}{a \bar{X}+b}\right) g \rho_{y x} C_{y} C_{x}\right] \\
\operatorname{Bias}\left(\bar{y}_{C S}^{*}\right)=\lambda \bar{Y}\left[(2 \alpha-1) g \rho_{y x} C_{y} C_{x}+\alpha g^{2} C_{x}^{2}\right] \\
\operatorname{Bias}\left(\bar{y}_{R d}^{*}\right)=-\lambda g \bar{Y} \rho_{y x} C_{y} C_{x} \\
M S E\left(\bar{y}_{P R}^{*}\right)=\lambda \bar{Y}^{2}\left[C_{y}^{2}+\left\{\frac{a \bar{X}}{a \bar{X}+b}\right\}^{2} g^{2}(2 \eta-1)^{2} C_{x}^{2}+\frac{a \bar{X}}{a \bar{X}+b} g(2 \eta-1) \rho_{y x} C_{y} C_{x}\right] \\
M S E\left(\bar{y}_{C S}^{*}\right)=\bar{Y}^{2} \lambda\left[C_{y}^{2}+g(2 \alpha-1) C_{x}^{2}\left\{g(2 \eta-1)+\rho_{y x} \frac{C_{y}}{C_{x}}\right\}\right] \\
M S E\left(\bar{y}_{R d}^{*}\right)=\lambda\left[\bar{Y}^{2} C_{y}^{2}-2 g \bar{Y} \rho_{y x} C_{x} C_{y}(\bar{Y}-\alpha \bar{X})+g C_{x}^{2}(\bar{Y}-\alpha \bar{X})^{2}\right]
\end{gathered}
$$


Furthermore, the minimum attainable MSEs of the estimators $\bar{y}_{P R}^{*}, \bar{y}_{C S}^{*}$ and $\bar{y}_{R d}^{*}$ are

$$
\begin{aligned}
& \operatorname{MSE}\left(\bar{y}_{P R}^{*}\right)_{\min }=\lambda \bar{Y}^{2} C_{y}^{2}\left(1-\rho_{y x}^{2}\right) \\
& \operatorname{MSE}\left(\bar{y}_{C S}^{*}\right)_{\min }=\lambda \bar{Y}^{2} C_{y}^{2}\left(1-\rho_{y x}^{2}\right) \\
& \operatorname{MSE}\left(\bar{y}_{R d}^{*}\right)_{\min }=\lambda \bar{Y}^{2} C_{y}^{2}\left(1-\rho_{y x}^{2}\right)
\end{aligned}
$$

Hence, we have

$$
M S E\left(\bar{y}_{P R}^{*}\right)_{\min }=\operatorname{MSE}\left(\bar{y}_{C S}^{*}\right)_{\min }=\operatorname{MSE}\left(\bar{y}_{R d}^{*}\right)_{\min }=\lambda \bar{Y}^{2} C_{y}^{2}\left(1-\rho_{y x}^{2}\right)
$$

Table 1 and Eqs. (19) to (21) and Eq. (25) substantiate that the modified ratio and product type estimators are too complex in structure, demands advance knowledge of the scalars and the minimum MSEs of these estimators are equivalent to the MSE of linear regression estimator $\hat{\bar{Y}}_{\text {reg }}$ as given in Eq. (9). Thus, making their theoretical and practical relevance in the argument.

\section{Proposed Estimator}

We define an efficient variant of dual to product and ratio estimators for $\bar{Y}$ as

$$
\hat{\bar{Y}}_{M d}=\bar{y}\left(\frac{\bar{X}+\theta \bar{x}^{*}}{\bar{x}^{*}+\theta \bar{X}}\right)
$$

where $\theta$ is a scalar which is determined so as to minimize the $M S E$ of the proposed

estimator $\hat{\bar{Y}}_{M d}$. Also, it is worth noting that, for $\theta=1, \hat{\bar{Y}}_{M d}=\bar{y}$ and that, for $\theta=0, \hat{\bar{Y}}_{M d}=\bar{y}_{P}^{*}$. Moreover, if $\theta$ is very large, $\hat{\bar{Y}}_{M d}$ is almost the same as $\bar{y}_{R}^{*}$.

The Bias and mean square error (MSE) of the proposed estimator $\hat{\bar{Y}}_{M d}$ are obtained by considering

such that $E\left(e_{0}\right)=E\left(e_{1}\right)=0$.

$$
\bar{y}=\bar{Y}\left(1+e_{0}\right), \bar{x}=\bar{X}\left(1+e_{1}\right)
$$

Also, on simplification, we get

$$
E\left(e_{0}^{2}\right)=\lambda C_{y}^{2}, E\left(e_{1}^{2}\right)=\lambda C_{x}^{2}, E\left(e_{0} e_{1}\right)=\lambda \rho_{y x} C_{y} C_{x}
$$

Now, expressing Eq. 26) in terms of $e_{0}, e_{1}$ we get

$$
\hat{\bar{Y}}_{M d}=\bar{Y}\left(1+e_{0}\right)\left\{1-\frac{\theta g e_{1}}{(1+\theta)}\right\}\left\{1-\frac{g e_{1}}{(1+\theta)}\right\}^{-1}
$$

Expanding the right hand side of Eq. 28), multiplying out, and retaining the terms up to second powers of $e$ 's, we get 
or

$$
\hat{\bar{Y}}_{M d}=\bar{Y}\left\{1+e_{0}+\frac{(1-\theta)}{(1+\theta)} g e_{1}+\frac{(1-\theta)}{(1+\theta)} g e_{0} e_{1}+\frac{(1-\theta)}{(1+\theta)^{2}} g^{2} e_{1}^{2}\right\}
$$

$$
\hat{\bar{Y}}_{M d}-\bar{Y}=\bar{Y}\left\{e_{0}+\frac{(1-\theta)}{(1+\theta)} g e_{1}+\frac{(1-\theta)}{(1+\theta)} g e_{0} e_{1}+\frac{(1-\theta)}{(1+\theta)^{2}} g^{2} e_{1}^{2}\right\}
$$

Taking the expectation in Eq. (30) and using results in Eq. (27), we get the bias of $\hat{\bar{Y}}_{M d}$ to the first degree of approximation as

$$
\operatorname{Bias}\left(\hat{\bar{Y}}_{M d}\right)=\lambda \bar{Y}\left\{\frac{(1-\theta)}{(1+\theta)} g \rho_{y x} C_{y} C_{x}+\frac{(1-\theta)}{(1+\theta)^{2}} g^{2} C_{x}^{2}\right\}
$$

Again from Eq. (30), by neglecting the terms of $e$ 's having degree greater than one, we have

$$
\hat{\bar{Y}}_{M d}-\bar{Y}=\bar{Y}\left[e_{0}+\left(\frac{1-\theta}{1+\theta}\right) g e_{1}\right]
$$

Squaring both sides of Eq. (32), taking the expectation and using results in Eq. 27), we obtain the $M S E$ of $\hat{\bar{Y}}_{M d}$ to the first degree of approximation as

$$
\operatorname{MSE}\left(\hat{\bar{Y}}_{M d}\right)=\lambda \bar{Y}^{2}\left[C_{y}^{2}+\left(\frac{1-\theta}{1+\theta}\right)^{2} g^{2} C_{x}^{2}+2 g\left(\frac{1-\theta}{1+\theta}\right) \rho_{y x} C_{y} C_{x}\right]
$$

Minimization of $M S E\left(\hat{\bar{Y}}_{M d}\right)$ in Eq. 33 with respect to $\theta$ yields the optimum value of $\theta$ as

$$
\theta_{\text {opt }}=\frac{g+\rho_{y x} \frac{C_{y}}{C_{x}}}{g-\rho_{y x} \frac{C_{y}}{C_{x}}}
$$

On substituting Eq. 34 in Eq. (33), the minimum attainable $M S E$ of $\hat{\bar{Y}}_{M d}$ is obtained as

$$
\operatorname{MSE}\left(\hat{\bar{Y}}_{M d}\right)_{\min }=\lambda \bar{Y}^{2} C_{y}^{2}\left(1-\rho_{y x}^{2}\right)
$$

Remark 1. The minimum MSE of $\hat{\bar{Y}}_{M d}$ is same as that of the MSE of the linear regression estimator $\hat{\bar{Y}}_{\text {reg }}$ as given in Eq. (9).

Even our proposed estimator's minimum $M S E$ corroborate the results of the other modified ratio and product type estimators' minimum MSEs as given in Eq. (25). But now we will work out a simple condition on our proposed estimator in order to derive a new proposed estimator for which previously proposed estimator will 
be called parent estimator. Hence, using the parent estimator our new proposed estimator is

$$
\hat{\bar{Y}}_{w}=w \hat{\bar{Y}}_{M d}
$$

where $w$ denotes the scalar which is to be suitably determined so as to minimize the $M S E$ of the above concerned estimator.

Now, expanding Eq. (36) using Eq. (29), we get

or

$$
\hat{\bar{Y}}_{w}=w \bar{Y}\left\{1+e_{0}+\frac{(1-\theta)}{(1+\theta)} g e_{1}+\frac{(1-\theta)}{(1+\theta)} g e_{0} e_{1}+\frac{(1-\theta)}{(1+\theta)^{2}} g^{2} e_{1}^{2}\right\}
$$

$$
\hat{\bar{Y}}_{w}-\bar{Y}=(w-1) \bar{Y}+w \bar{Y}\left\{e_{0}+\frac{(1-\theta)}{(1+\theta)} g e_{1}+\frac{(1-\theta)}{(1+\theta)} g e_{0} e_{1}+\frac{(1-\theta)}{(1+\theta)^{2}} g^{2} e_{1}^{2}\right\}
$$

Squaring both sides of Eq. (38), taking the expectation and using results in Eq. 27), we obtain the $M S E$ of $\bar{Y}_{w}$ to the first degree of approximation as

$$
\begin{gathered}
\operatorname{MSE}\left(\hat{\bar{Y}}_{w}\right)=(w-1)^{2} \bar{Y}^{2}+w^{2} \lambda \bar{Y}^{2}\left\{C_{y}^{2}+\left(\frac{1-\theta}{1+\theta}\right)^{2} g^{2} C_{x}^{2}+2 g\left(\frac{1-\theta}{1+\theta}\right) \rho_{y x} C_{y} C_{x}\right\} \\
+2 w(w-1) \lambda \bar{Y}\left\{\frac{1-\theta}{1+\theta} g \rho_{y x} C_{y} C_{x}+\frac{1-\theta}{(1+\theta)^{2}} g^{2} C_{x}^{2}\right\}
\end{gathered}
$$

which can be rewritten as

$$
\operatorname{MSE}\left(\hat{\bar{Y}}_{w}\right)=(w-1)^{2} \bar{Y}^{2}+w^{2} M S E\left(\hat{\bar{Y}}_{M d}\right)+2 w(w-1) \bar{Y} \operatorname{Bias}\left(\hat{\bar{Y}}_{M d}\right)
$$

From Eq. 40 it can be brought to notice that the $M S E$ of the new proposed estimator contains the MSE and Bias of its parent estimator. Now differentiating Eq. 40 w.r.t $w$ and equating it to zero, we get

$$
w_{\text {opt }}=\frac{\bar{Y}^{2}+\bar{Y} \operatorname{Bias}\left(\hat{\bar{Y}}_{M d}\right)}{\bar{Y}^{2}+M S E\left(\hat{\bar{Y}}_{M d}\right)+2 \bar{Y}_{\operatorname{Bias}\left(\hat{\bar{Y}}_{M d}\right)}}
$$

and using it to find the minimum $M S E$ of the new proposed estimator, we have

$$
\operatorname{MSE}\left(\hat{\bar{Y}}_{w}\right)_{\min }=\frac{\bar{Y}^{2}\left(\operatorname{MSE}\left(\hat{\bar{Y}}_{M d}\right)-\operatorname{Bias}\left(\hat{\bar{Y}}_{M d}\right)^{2}\right)}{\bar{Y}^{2}+M S E\left(\hat{\bar{Y}}_{M d}\right)+2 \bar{Y} \operatorname{Bias}\left(\hat{\bar{Y}}_{M d}\right)}
$$

From Eq. (42), we see that the numerator is nothing but variance of the parent estimator. The trade-off between bias and variance in order to increase the efficiency of the new proposed estimator is very effective here. 
If we substitute in equation Eq. 42, the minimum attainable $M S E$ of parent estimator $\hat{\bar{Y}}_{M d}$, we get

$$
\operatorname{MSE}\left(\hat{\bar{Y}}_{w}\right)_{\min }=\frac{\bar{Y}^{2}\left(M S E\left(\hat{\bar{Y}}_{r e g}\right)-B^{2}\right)}{\bar{Y}^{2}+M S E\left(\hat{\bar{Y}}_{r e g}\right)+2 \bar{Y} B}
$$

where $B$ represents the $\operatorname{Bias}\left(\hat{\bar{Y}}_{M d}\right)$ at the value of $\theta=\theta_{\text {opt }}$ as given in Eq. (34). That is

$$
B=-\frac{\lambda \bar{Y}}{2}\left\{g \rho_{y x} C_{y} C_{x}+\rho_{y x}^{2} C_{x}^{2}\right\}
$$

Theorem 1. For small sample size, the proposed estimator $\hat{\bar{Y}}_{w}$ is more efficient than the regression estimator $\hat{\bar{Y}}_{\text {reg. }}$. But as the sample size increases, i.e., as $n \rightarrow N$ the relative efficiency of the proposed estimator $\hat{\bar{Y}}_{w}$ is same as that of the regression estimator $\hat{\bar{Y}}_{\text {reg }}$.

Proof. From the definition of relative efficiency $R E$, we get:

$$
R E=\frac{M S E\left(\hat{\bar{Y}}_{\text {reg }}\right)}{M S E\left(\hat{\bar{Y}}_{w}\right)_{\text {min }}}=\frac{1}{\bar{Y}^{2}\left(1-\frac{B^{2}}{M S E\left(\hat{Y}_{r e g}\right)}\right)}\left(\bar{Y}^{2}+\operatorname{MSE}\left(\hat{\bar{Y}}_{r e g}\right)+2 \bar{Y} B\right)
$$

Now as $n \rightarrow N$ we have $\lambda \rightarrow 0$. As a result $\frac{B^{2}}{M S E\left(\hat{Y}_{\text {reg }}\right)} \rightarrow 0, M S E\left(\hat{\bar{Y}}_{\text {reg }}\right) \rightarrow 0$ and $B \rightarrow 0$. Therefore $R E \rightarrow 1$
i.e., $\operatorname{MSE}\left(\hat{\bar{Y}}_{w}\right)_{\text {min }} \rightarrow \operatorname{MSE}\left(\hat{\bar{Y}}_{\text {reg }}\right)$. Hence the theorem.

\section{Bias and EfFiciency Comparisons}

It is well known that Bias and MSE of the usual unbiased estimator $\bar{y}$ for population mean in SRSWOR are

$$
\begin{gathered}
\operatorname{Bias}(\bar{y})=0 \\
V(\bar{y})=\lambda \bar{Y}^{2} C_{y}{ }^{2}
\end{gathered}
$$

For making Bias comparisons of the proposed estimator $\hat{\bar{Y}}_{M d}$ with the existing estimators, we have from Eq. (31), and Eq. (3), Eq. (4), Eq. (12), Eq. (13), Eq. (16), Eq. 17), Eq. 18), and Eq. 45.

(i) $\left|\operatorname{Bias}\left(\hat{\bar{Y}}_{M d}\right)\right| \leq|B(\bar{y})|$ or $\left|\operatorname{Bias}\left(\hat{\bar{Y}}_{M d}\right)\right| \leq 0$ if

$$
\theta=0
$$


AN EFFICIENT VARIANT OF DUAL TO PRODUCT AND RATIO ESTIMATORS IN SAMPLE SURVEYS

(ii) $\left|\operatorname{Bias}\left(\hat{\bar{Y}}_{M d}\right)\right| \leq\left|\operatorname{Bias}\left(\bar{y}_{R}\right)\right|$ if

$$
\left[\frac{g^{2}(1-\theta)^{2}\left(g C_{x}+\rho_{y x} C_{y}(1+\theta)\right)^{2}}{(1+\theta)^{4}}-\left(C_{x}-\rho_{y x} C_{y}\right)^{2}\right] \leq 0
$$

(iii) $\left|\operatorname{Bias}\left(\hat{\bar{Y}}_{M d}\right)\right| \leq\left|\operatorname{Bias}\left(\bar{y}_{P}\right)\right|$ if

$$
\left[\frac{g^{2}(1-\theta)^{2}\left(g C_{x}+\rho_{y x} C_{y}(1+\theta)\right)^{2}}{(1+\theta)^{4}}-\left(\rho_{y x} C_{y}\right)^{2}\right] \leq 0
$$

(iv) $\left|\operatorname{Bias}\left(\hat{\bar{Y}}_{M d}\right)\right| \leq\left|\operatorname{bias}\left(\bar{y}_{R}^{*}\right)\right|$ if

$$
\left[\frac{(1-\theta)^{2}\left(g C_{x}+\rho_{y x} C_{y}(1+\theta)\right)^{2}}{(1+\theta)^{4}}-\left(\rho_{y x} C_{y}\right)^{2}\right] \leq 0
$$

(v) $\left|\operatorname{Bias}\left(\hat{\bar{Y}}_{M d}\right)\right| \leq\left|\operatorname{Bias}\left(\bar{y}_{P}^{*}\right)\right|$ if

$$
\left[\frac{g^{2}(1-\theta)^{2}\left(g C_{x}+\rho_{y x} C_{y}(1+\theta)\right)^{2}}{(1+\theta)^{4}}-\left(C_{x}+g \rho_{y x} C_{y}\right)^{2}\right] \leq 0
$$

(vi) $\left|\operatorname{Bias}\left(\hat{\bar{Y}}_{M d}\right)\right| \leq\left|\operatorname{Bias}\left(\bar{y}_{P R}^{*}\right)\right|$ if

$$
\begin{gathered}
{\left[\frac{(1-\theta)^{2}\left(g C_{x}+\rho_{y x} C_{y}(1+\theta)\right)^{2}}{(1+\theta)^{4}}\right.} \\
\left.-\left(\frac{a \bar{X}}{a \bar{X}+b}\right)^{2}\left(g \eta\left(\frac{a \bar{X}}{a \bar{X}+b}\right) C_{x}-(1-2 \eta) \rho_{y x} C_{y}\right)^{2}\right] \leq 0
\end{gathered}
$$

(vii) $\left|\operatorname{Bias}\left(\hat{\bar{Y}}_{M d}\right)\right| \leq\left|\operatorname{Bias}\left(\bar{y}_{C S}^{*}\right)\right|$ if

$$
\left[\frac{(1-\theta)^{2}\left(g C_{x}+\rho_{y x} C_{y}(1+\theta)\right)^{2}}{(1+\theta)^{4}}-\left(g \alpha C_{x}-(1-2 \alpha) \rho_{y x} C_{y}\right)^{2}\right] \leq 0
$$

(viii) $\left|\operatorname{Bias}\left(\hat{\bar{Y}}_{M d}\right)\right| \leq\left|\operatorname{Bias}\left(\bar{y}_{R d}^{*}\right)\right|$ if

$$
\left[\frac{(1-\theta)^{2}\left(g C_{x}+\rho_{y x} C_{y}(1+\theta)\right)^{2}}{(1+\theta)^{4}}-\left(\rho_{y x} C_{y}\right)^{2}\right] \leq 0
$$

For making efficiency comparisons of the proposed estimator $\hat{\bar{Y}}_{M d}$ with the existing estimators, we have from Eq. (33), and Eq. (5), Eq. (6), Eq. (14), Eq. (15), and 46 )

(i) $M S E\left(\hat{\bar{Y}}_{M d}\right)<V(\bar{y})$ if

$$
\min \left(0,-2 \rho_{y x} \frac{C_{y}}{g C_{x}}\right)<\psi<\max \left(0,-2 \rho_{y x} \frac{C_{y}}{g C_{x}}\right)
$$


where $\psi=\frac{1-\theta}{1+\theta}$.

(ii) $M S E\left(\hat{\bar{Y}}_{M d}\right)<M S E\left(\bar{y}_{R}\right)$ if

$\min \left\{-\frac{1}{g},\left(-2 \rho_{y x} \frac{C_{y}}{g C_{x}}+\frac{1}{g}\right)\right\}<\psi<\max \left\{-\frac{1}{g},\left(-2 \rho_{y x} \frac{C_{y}}{g C_{x}}+\frac{1}{g}\right)\right\}$

(iii) $\operatorname{MSE}\left(\hat{\bar{Y}}_{M d}\right)<M S E\left(\bar{y}_{P}\right)$ if

$$
\min \left\{\frac{1}{g},\left(-2 \rho_{y x} \frac{C_{y}}{g C_{x}}-\frac{1}{g}\right)\right\}<\psi<\max \left\{\frac{1}{g},\left(-2 \rho_{y x} \frac{C_{y}}{g C_{x}}-\frac{1}{g}\right)\right\}
$$

(iv) $\operatorname{MSE}\left(\hat{\bar{Y}}_{M d}\right)<M S E\left(\bar{y}_{R}^{*}\right)$ if

$$
-1<\psi<\left(1-2 \rho_{y x} \frac{C_{y}}{g C_{x}}\right)
$$

(v) $\operatorname{MSE}\left(\hat{\bar{Y}}_{M d}\right)<\operatorname{MSE}\left(\bar{y}_{P}^{*}\right)$ if

$$
\min \left\{1,\left(-2 \rho_{y x} \frac{C_{y}}{g C_{x}}-1\right)\right\}<\psi<\max \left\{1,\left(-2 \rho_{y x} \frac{C_{y}}{g C_{x}}-1\right)\right\}
$$

Now let us denote the estimators $\hat{\bar{Y}}_{M d}, \bar{y}_{P R}^{*}, \bar{y}_{C S}^{*}$ and $\bar{y}_{R d}^{*}$ which attains minimum MSEs equivalent to MSE of linear regression estimator $\hat{\bar{Y}}_{\text {reg }}$ as $T$, and comparing it to the new proposed estimator $\hat{\bar{Y}}_{w}$, we have

(vi) $M S E\left(\hat{\bar{Y}}_{w}\right)_{\min }<M S E(T)$ if

$$
\begin{gathered}
(M S E(T)+\bar{Y} B)^{2}>0 \\
\text { where } M S E(T)=M S E\left(\hat{\bar{Y}}_{\text {reg }}\right)=\lambda \bar{Y}^{2} C_{y}^{2}\left(1-\rho_{y x}^{2}\right)
\end{gathered}
$$

\section{EMPirical Study}

To examine the merits of the new proposed estimator $\hat{\bar{Y}}_{w}$ over other existing estimators, seven natural population data sets have been considered. The description of the populations and the values of various parameters are listed in Tables 2 and 3. respectively.

In Table 4, the effective ranges of $\psi$ along with its optimum values are shown for which the proposed estimator $\hat{\bar{Y}}_{M d}$ is better than the other existing estimators. However, in practice, it may be difficult to determine the interval extremes depending on the unknown parameter values of the population. 
The percentage relative efficiencies (PREs) are obtained for various suggested estimators of $\bar{Y}$ with respect to the usual unbiased estimator $\bar{y}$ using the formula

$$
\operatorname{PRE}(\phi, \bar{y})=\frac{V(\bar{y})}{M S E(\phi)} \times 100
$$

where $\phi$ is used in places of any estimator among $\bar{y}, \bar{y}_{R}, \bar{y}_{P}, \bar{y}_{R}^{*}, \bar{y}_{P}^{*}, \hat{\bar{Y}}_{M d}$ and $\hat{\bar{Y}}_{w}$, and the findings are presented in Table 5

TABle 2. Description of Populations

\begin{tabular}{|c|c|}
\hline Populations & Variables \\
\hline \multirow{3}{*}{$\begin{array}{l}\text { Population I } \\
\text { Kadilar and Cingi } 9\end{array}$} & $Y=$ Apple production amount in 1999 \\
\hline & $X=$ Apple production amount in 1998 \\
\hline & $N=204, n=50$ \\
\hline \multirow{3}{*}{$\begin{array}{l}\text { Population II } \\
\text { Sukhatme and Chand } 16\end{array}$} & $Y=$ Apple trees of bearing age in 1964 \\
\hline & $X=$ Bushels of apples harvested in 1964 \\
\hline & $N=200, n=20$ \\
\hline \multirow{3}{*}{$\begin{array}{l}\text { Population III } \\
\text { Cochran } 5\end{array}$} & $Y=$ Peach production in bushels in an orchard in 1946 \\
\hline & $X=$ Number of peach trees in the orchard in 1946 \\
\hline & $N=256, n=100$ \\
\hline \multirow{3}{*}{$\begin{array}{l}\text { Population IV } \\
\text { Singh } 14\end{array}$} & $Y=$ Number of females employed \\
\hline & $X=$ Number of females in service \\
\hline & $N=61, n=20$ \\
\hline \multirow{3}{*}{$\begin{array}{l}\text { Population V } \\
\text { Das } 6\end{array}$} & $Y=$ Number of agricultural laborers for 1971 \\
\hline & $X=$ Number of agricultural laborers for 1961 \\
\hline & $N=278, n=30$ \\
\hline \multirow{3}{*}{$\begin{array}{l}\text { Population VI } \\
\text { Maddala } 10\end{array}$} & $Y=$ Consumption per capita \\
\hline & $X=$ Deflated prices of veal \\
\hline & $N=16, n=4$ \\
\hline \multirow{3}{*}{$\begin{array}{l}\text { Population VII } \\
\text { Johnston } 8\end{array}$} & $Y=$ Percentage of hives affected by disease \\
\hline & $\begin{array}{l}X=\text { Date of flowering of a particular summer species } \\
\text { (number of days from January } 1 \text { ) }\end{array}$ \\
\hline & $N=10, n=4$ \\
\hline
\end{tabular}


TABle 3. Parameters of populations

\begin{tabular}{|c|cccccc|}
\hline Populations & $\bar{Y}$ & $\bar{X}$ & $C_{y}$ & $C_{x}$ & $\rho_{y x}$ & $g$ \\
\hline \hline I & 966 & 1014 & 2.4739 & 2.4866 & 0.94 & 0.3247 \\
\hline II & 1031.82 & 2934.58 & 1.5977 & 2.0062 & 0.93 & 0.1111 \\
\hline III & 56.47 & 44.45 & 1.42 & 1.40 & 0.887 & 0.6410 \\
\hline IV & 7.46 & 5.31 & 0.7103 & 0.7574 & 0.7737 & 0.4878 \\
\hline V & 39.0680 & 25.1110 & 1.4451 & 1.6198 & 0.7213 & 0.1209 \\
\hline VI & 7.6375 & 75.4313 & 0.2278 & 0.0986 & -0.6823 & 0.3333 \\
\hline VII & 52 & 200 & 0.1562 & 0.0458 & -0.94 & 0.6667 \\
\hline
\end{tabular}

TABLE 4. Effective ranges of $\psi$ under which $\hat{\bar{Y}}_{M d}$ is better than the other existing estimators

\begin{tabular}{ccccccc}
\hline Population & \multicolumn{5}{c}{ Range of $\psi$ in which $\hat{\underline{Y}}_{M d}$ is better than } & \multicolumn{2}{c}{$\begin{array}{c}\text { Optimum value of } \psi \\
\left(\text { i.e., } \psi_{0}\right)\end{array}$} \\
\cline { 2 - 6 } & $\bar{y}$ & $\bar{y}_{R}$ & $\bar{y}_{P}$ & $\bar{y}_{R}^{*}$ & $\bar{y}_{P}^{*}$ & -2.8804 \\
\hline \hline I & $(-5.7608,0)$ & $(-3.08,-2.6808)$ & $(-8.8408,3.08)$ & $(-4.7608,-1)$ & $(-6.7608,1)$ & -6.6657 \\
\hline II & $(-13.3315,0)$ & $(-9,-4.3315)$ & $(-22.3315,9)$ & $(-12.3315,-1)$ & $(-14.3315,1)$ & -1.4035 \\
\hline III & $(-2.8069,0)$ & $(-1.56,-1.2469)$ & $(-4.3669,1.56)$ & $(-1.8069,-1)$ & $(-3.8069,1)$ & -1.4875 \\
\hline IV & $(-2.975,0)$ & $(-2.05,-0.9250)$ & $(-5.025,2.05)$ & $(-1.975,-1)$ & $(-3.975,1)$ & -5.3226 \\
\hline V & $(-10.6452,0)$ & $(-8.2713,-2.3739)$ & $(-18.9165,8.2713)$ & $(-9.6452,-1)$ & $(-11.6452,1)$ & 4.7299 \\
\hline VI & $(0,9.4598)$ & $(-3.0003,12.4601)$ & $(3.0003,6.4595)$ & $(-1,10.4598)$ & $(1,8.4598)$ & 4.8062 \\
\hline VII & $(0,9.6125)$ & $(-1.5,11.1125)$ & $(1.5,8.1125)$ & $(-1,10.6125)$ & $(1,8.6125)$ & \\
\hline
\end{tabular}

\section{Discussion And Conclusion}

Section 3 examines how, within a very wide range of $\psi$, the proposed estimator $\hat{\bar{Y}}_{M d}$ behaves more efficiently than the other estimators namely $\bar{y}, \bar{y}_{R}, \bar{y}_{P}, \bar{y}_{R}^{*}$ and $\bar{y}_{P}^{*}$. Table 4 provides the effective ranges of $\psi$ along with its optimum values for which the proposed estimator $\hat{\bar{Y}}_{M d}$ is more efficient than the other existing estimators as far as the $M S E$ criterion is considered. In section 2 we see that $M S E$ of the estimator $\hat{\bar{Y}}_{M d}$ is equivalent to the $M S E$ of $\hat{\bar{Y}}_{\text {reg }}$. But using the procedure to lower the $M S E$ and forming the new proposed estimator by simply conditioning the parent estimator, we obtain a more efficient estimator than the linear regression estimator. The two estimators (linear regression estimator and new proposed estimator) needs an equal number of prior knowledge of population parameters $\left(S_{y}\right.$ and $\left.S_{x}\right)$ but the reason why the latter is more efficient is it utilizes the knowledge of Bias of 
TABle 5. Percentage Relative Efficiencies (PREs) of various estimators with respect to $\bar{y}$

\begin{tabular}{cccccccc}
\hline \multirow{2}{*}{ Estimators } & \multicolumn{7}{c}{ Populations } \\
\cline { 2 - 8 } & $\mathrm{I}$ & $\mathrm{II}$ & $\mathrm{III}$ & $\mathrm{IV}$ & $\mathrm{V}$ & $\mathrm{VI}$ & $\mathrm{VII}$ \\
\hline \hline $\bar{y}$ & 100 & 100 & 100 & 100 & 100 & 100 & 100 \\
\hline $\bar{y}_{R}$ & 828.89 & 414.66 & 448.4 & 205.35 & 156.39 & $*$ & $*$ \\
\hline $\bar{y}_{P}$ & $*$ & $*$ & $*$ & $*$ & $*$ & 167.58 & 187.08 \\
\hline $\bar{y}_{R}^{*}$ & 202.85 & 131.59 & 359.38 & 214.74 & 121.53 & $*$ & $*$ \\
\hline $\bar{y}_{P}^{*}$ & $*$ & $*$ & $*$ & $*$ & $*$ & 121.37 & 149.13 \\
\hline$\hat{\bar{Y}}_{M d}$ & 859.11 & 740.19 & 468.98 & 249.14 & 208.45 & 187.10 & 859.11 \\
\hline$\hat{\bar{Y}}_{w}$ & 1076.6 & 844.75 & 474.85 & 249.24 & 209.24 & 187.52 & 868.68 \\
\hline
\end{tabular}

* Data is not applicable.

parent population. This is an additional work to see how different estimators with different Bias will affect the MSEs, which is a research question and is left by the authors for further work. In addition, our theoretical results is supported numerically based on the results obtained in Table 5 using the data sets as shown in Table 2 along with the required values of various parameters in Table 3 . Table 5 exhibits that there is a considerable gain in efficiency by using proposed estimator $\hat{\bar{Y}}_{w}$ over the estimators $\bar{y}, \bar{y}_{R}, \bar{y}_{P}, \bar{y}_{R}^{*}, \bar{y}_{P}^{*}$, and $\hat{\bar{Y}}_{M d}$. Thus, the new proposed estimator is more appropriate, in comparison to all the other existing estimators, for estimating the unknown mean $\bar{Y}$ of the study variable $Y$. Hence, the proposed estimator $\hat{\bar{Y}}_{w}$ should be preferred in practice. The present study deals with the estimation of unknown mean $\bar{Y}$ under SRSWOR scheme. It can also be extended to double (or two-phase) sampling, two-stage sampling and other sampling designs.

Author Contribution Statements The authors contributed equally. All authors read and approved the final copy of the manuscript.

Declaration of Competing Interests The authors declare that they have no known competing financial interests or personal relationships that could have appeared to influence the work reported in this paper.

Acknowledgments The authors are thankful to the Editor-in-Chief, Section Editor, and learned reviewers for their valuable comments towards the improvements of the manuscript. 


\section{REFERENCES}

[1] Adebola, F. B., Adegoke, N. A., Sanusi, R. A., A class of regression estimator with cum-dual ratio estimator as intercept, International Journal of Probability and Statistics, 4(2) (2015), 42-50. https://doi.org/10.5923/j.ijps.20150402.02

[2] Bandyopadhyaya, S., Improved ratio and product estimators, Sankhya: The Indian Journal of Statistics, series C, 42 (1980), 45-49.

[3] Choudhury, S., Singh, B. K., An efficient class of dual to product-cum-dual to ratio estimators of finite population mean in sample surveys, Global Journal of Science Frontier Research, 12(3) (2012), 25-33.

[4] Cochran, W. G., The estimation of the yields of cereal experiments by sampling for the ratio of grain to total produce, The Journal of Agricultural Science, 30(2) (1940), 262-275. https://doi.org/10.1017/S0021859600048012

[5] Cochran, W. G., Sampling Techniques, 3rd edition, John Wiley and Sons, New York, 1977.

[6] Das, A. K., Contribution to the theory of sampling strategies based on auxiliary information, Ph.D. thesis submitted to BCKV, Mohanpur, Nadia, West-Bengal, India, 1988.

[7] Enang, E. I., Uket, J. O., Ekpenyong, E. J., A modified class of exponential-type estimator of population mean in simple random sampling, International Journal of Advanced Statistics and Probability, 5(2) (2017), 70-76. https://doi.org/10.14419/ijasp.v5i2.7345

[8] Johnston, J., Econometric Methods, 2nd edition, Mc Graw Hill Book Company, Tokyo, 1972.

[9] Kadilar, C., Cingi, H., A new estimator using two auxiliary variables, Applied Mathematics and Computation, 162 (2005), 901-908. https://doi.org/10.1016/j.amc.2003.12.130

[10] Maddala, G. S., Econometrics, Mc Graw Hill, New York, 1977.

[11] Murthy, M. N., Product method of estimation, Sankhya: The Indian Journal of Statistics, Series A, 26 (1964), 69-74.

[12] Robson, D. S., Application of multivariate polykays to the theory of unbiased ratiotype estimation, Journal of the American Statistical Association, 52 (1957), 511-522. https://doi.org/10.1080/01621459.1957.10501407

[13] Singh, H. P., Pal, S. K., Mehta, V., A generalized class of dual to product-cum-dual to ratio type estimators of finite population mean in sample surveys, Applied Mathematics \& Information Sciences Letters, 4(1) (2016), 25-33.

[14] Singh, M. P., Comparison of some ratio-cum-product estimators, Sankhya: The Indian Journal of Statistics, Series B, 31 (1969), 375-378.

[15] Srivenkataramana, T., A dual to ratio estimator in sample surveys, Biometrika, 67(1) (1980), 199-204. https://doi.org/10.1093/biomet/67.1.199

[16] Sukhatme, B. V., Chand, L., Multivariate ratio-type estimators, Proceedings of the Social Statistics Section of the American Statistical Association, (1977), 927-931. 